\title{
Diagnostic Vision and Association with Demographics in Malaria Patients
}

\author{
Ranweer $^{1}$, Jyotsna Chandwani ${ }^{*}$, Seema Gupta ${ }^{2}$, Geeta Parihar $^{1}$ and Geeta Pachori ${ }^{2}$ \\ ${ }^{1}$ Department of Microbiology, ${ }^{2}$ Department of Pathology, JLN, MC, Ajmer, Rajasthan, India \\ *Corresponding author
}

\section{A B S T R A C T}

Since many years malaria is a scourge of mankind. According to the report, there were 212 million new cases and 429000 malaria deaths worldwide in 2015. India contributes $70 \%$ of malaria cases in the South-East Asia region. This study was conducted as there were only a few studies available in central part of Rajasthan. The main objective is to study the

Keywords

Malaria, $P$. vivax, $P$. falciparum, Diagnostic

\section{Article Info}

Accepted: 06 December 2017 Available Online: 10 January 2018 demographic profile of malaria cases and to have a diagnostic vision on them. This was a laboratory based observational, descriptive and retrospective type of study, conducted in Jawahar Lal Nehru Medical College, Ajmer. The data was collected from 1 April2016 to 31 march2017. Statistical analysis was performed with the SPSS, Trial version 23 for Windows statistical software package (SPSS inc., Chicago, il, USA) and Primer. A total 27,455patients were tested by rapid diagnostic method and 3810 slides were examined. Out of which 502 and 79 were positive for malaria, respectively. A total of 530 malaria positive cases, $59.32 \%$ were male and $40.67 \%$ were female. Maximum number were found in age group 21 to 30 years and $<10$ years which was $23.77 \%$ and $22.08 \%$. A total number of cases were highest in September $(22.64 \%)$. In RDT out of 502 positive cases, $P$. vivax and $P$. falciparum were $54.91 \%$ and $39.81 \%$ were as in slide test. Out of 79 cases $79.74 \%$, $17.72 \%$ and $2.53 \%$ were $P$. vivax, $P$. falciparum and both respectively. Early diagnosis and control activities well ahead of the monsoon season should target more to the communities with lower socio-economic status and the young age groups.

\section{Introduction}

In India malaria was discovered by Sir, Ronald Ross in 1897. A century have passed, after its discovery, still malaria continues to be one of the leading public health problem in India (Estimation of True Malaria Burden in India).

Malaria is a mosquito-borne parasitic disease caused by several species of the Plasmodium parasite. Genus Plasmodium has five species, such as $P$. falciparum, $P$. vivax, $P$. ovale, $P$. malariae are human malaria species and $P$. knowlesi has been recorded recently causing malaria among monkeys in certain areas of South-East Asia. P. falciparum and P. Vivax malaria pose the greatest public health challenge. (Dharmendra Prasad Singh et al., 2016). Human malaria is transmitted by 60 species of female Anopheles mosquito. Out of 45 species of Anopheles mosquito in India, only few are regarded as vectors which are An. culicifacies, 
An. fluviatilis, An. stephensi, An. minimus, An. philippinensis, An. sundaicus, etc. (Paniker's textbook of medical parasitology).

Malaria life cycle passes its life in 2 host; definitive host is female Anopheles mosquito and intermediate host is Man. There are mainly 2 phases of development in human host.one is inside the liver which includes pre erythrocytic schizogony in which no clinical and no pathogenicity occur and hypnozoite stage which is the cause of relapse. Other is in $\mathrm{RBC}$ that is erythrocytic phase which includes erythrocytic schizogony that causes malarial paroxysm and gametogony that is an infect mosquito (Chatterjee et al., 2009).

The clinical manifestation in malaria involves a series of febrile paroxysms like cold stage, hot stage and sweating stage, followed by splenomegaly and anaemia (Chatterjee et al., 2009)

Malaria typically manifests as a febrile illness that can be fatal if unrecognized, especially in young children (Alireza Salimi Khorashad et al., 2014).

Microscopy is the reference/gold standard for the laboratory diagnosis of malaria parasite but its turnaround time is much more than that of RDT and it requires adequate training. RDTs are alternative diagnostic methods because they are quick and easy to carry out (Ruby Naz et al., 2016).

According to the report, there were 212 million new cases and 429000 malaria deaths worldwide in 2015. India contributes $70 \%$ of malaria cases in the South-East Asia region. This study was done as there are few studies available in central part of Rajasthan.

The Objective was to study the demographic profile of malaria cases and to have a diagnostic vision on them.

\section{Materials and Methods}

This study was carried out at Department of Microbiology, Pathology Laboratory Jawahar Lal Nehru Medical College, Ajmer, India, over a period of one year from 1 April 2016 to 31 march2017.

\section{Study design}

Observational, descriptive study.

\section{Study type}

Retrospective type of study.

\section{Statistical test}

Statistical analysis was performed with the SPSS, Trial version 23 for Windows statistical software package (SPSS inc., Chicago, il, USA) and Primer.

The Categorical data were presented as numbers (percent) and were compared among groups using Chi square test.

\section{Inclusion criteria}

A total 31,265 suspected malaria cases were subjected to smear microscopy or rapid diagnostic tests. (Rapid card Test=27455; Slide test=3810).

\section{Exclusion criteria}

Patients with other positive laboratory test results i.e. for typhoid fever and dengue fever.

Five $\mathrm{ml}$ blood ( $3 \mathrm{ml}$ in case of children) was collected through venipuncture by expert technician in full aseptic conditions. Peripheral thick and thin blood smears were prepared on same slide and stained with Leishmann stain as per standard laboratory protocol. 


\section{Microscopy (done in pathology lab)}

Leishmann stained blood films were examined under oil immersion fields. Stained slides were considered positive when at least one parasite was found, and negative if no parasite is found in 200 oil fields. Thin smear blood film was examined for species identification.

\section{Rapid diagnostic tests (done in microbiology lab)}

Commercially available ParaHIT Total ver.1.0 was used for detection malarial antigens such as HRP-2 antigen and Pan specific aldolase antigen.

\section{Principle}

Immuno-chromatography, in which nitrocellulose membrane is coated with antiHRP II antibody which is specific for $P$. falciparum and anti-aldolase antibody which detects the presence of any of Plasmodium species.

\section{Procedure}

Take $8 \mathrm{~mL}$ of sample with micropipette window A of test device Add 4 drops (200mL) of reaction buffer to window B of test device read the test at the end of 25 minutes.

\section{Results and Discussion}

A total 31,265 suspected malaria cases were subjected to smear microscopy or rapid diagnostic tests. (Rapid card Test=27455; Slide test $=3810$ ). Out of which 530 cases were positive for malaria.

No significant difference was observed in gender according to age group. Most of the cases were observed in 21 to years of age group as compared to extreme of age group. Males were more in all age group as compared to females in all age groups (Table 1).

There were 503 test positive for malaria through rapid diagnostic test, out of Pan (which is considered as Plasmodium vivax as there is no species existing in this area other than Plasmodium falciparum) were maximum that was 291(57.96\%) was found to be maximum followed by Plasmodium falciparum and mix. In case of slide test out of 79 positive cases again results were same Plasmodium vivax was maximum that were 63(79.74\%) (Fig. 1).

The maximum number of cases was more in August, September and October month (Fig. 2).

Specificity and PPV of rapid diagnostic test were $99.60 \%, 80.60 \%$ and sensitivity and NPV were $86.2 \%, 99 \%$ (Table 2).

Malaria is an important parasitic infection affecting large populations and continues to remain a serious public health problem in India.

In our study most of the cases were observed in 21 to30 years of age group followed by age group 1 to 10 years which was $23.78 \%$ and $22.08 \%$ respectively. Males were more in all age group as compared to females in all age groups.

Our study was similar with a study done in U.P by (Dharmendra Prasad Singh et al., (2016)) with a slight higher range affected in the above age groups that was $47.59 \%$ and $23.30 \%$ respectively and the number of males dominated over the females. Our study also coincided with a study done in Navi Mumbai by (Gurjeet Singh et al., (2015)). However, in a study conducted by EE Ayougu et al., (2016) in Nigeria $53(34.40 \%)$ were males while $101(65.50 \%)$ were females. 
Table.1 Baseline characteristics of the study population

\begin{tabular}{|c|c|c|}
\hline No of cases & 530 & \\
\hline Age Groups & No & $\%$ \\
\hline$<1$ Year & 9 & 1.69 \\
\hline 1TO 10 & 117 & 22.08 \\
\hline $11 \mathrm{~T} \mathrm{O20}$ & 94 & 17.73 \\
\hline 21 TO30 & 126 & 23.78 \\
\hline 31 T040 & 72 & 13.58 \\
\hline 41 TO 50 & 64 & 12.08 \\
\hline 51 TO60 & 20 & 3.78 \\
\hline 61 TO 70 & 16 & 3.01 \\
\hline$>70$ & 12 & 2.27 \\
\hline
\end{tabular}

\begin{tabular}{|c|c|c|c|c|c|}
\hline & \multicolumn{2}{|c|}{ F } & \multicolumn{2}{|c|}{$\mathbf{M}$} & \multirow[t]{2}{*}{ Total } \\
\hline & No & $\%$ & No & $\%$ & \\
\hline <1yEAR & 3 & 33.33 & 6 & 66.67 & 9 \\
\hline 1TO 10 & 47 & 40.17 & 70 & 59.83 & 117 \\
\hline $11 \mathrm{~T} 020$ & 29 & 30.85 & 65 & 69.15 & 94 \\
\hline 21 T030 & 48 & 38.10 & 78 & 61.90 & 126 \\
\hline 31 TO40 & 22 & 30.56 & 50 & 69.44 & 72 \\
\hline 41 TO 50 & 27 & 42.19 & 37 & 57.81 & 64 \\
\hline 51 ТО60 & 6 & 30.00 & 14 & 70.00 & 20 \\
\hline 61 TO 70 & 7 & 43.75 & 9 & 56.25 & 16 \\
\hline$>70$ & 6 & 50.00 & 6 & 50.00 & 12 \\
\hline & 195 & 36.79 & 335 & 63.21 & 530 \\
\hline
\end{tabular}

Chi-square $=5.775$ with 8 degrees of freedom; $\mathrm{P}=0.672 \mathrm{NS}$

Table.2 Evaluation of rapid malaria kit with microscopy

\begin{tabular}{|l|c|c|c|c|c|c|c|c|c|}
\hline & $\begin{array}{c}\text { Microscopy } \\
\text { positive }\end{array}$ & $\begin{array}{c}\text { Microscopy } \\
\text { negative }\end{array}$ & TOTAL & SN & SP & PPV & NPV & ACCURACY & P \\
\hline $\begin{array}{l}\text { Rapid } \\
\text { Reactive }\end{array}$ & 50 & 12 & 62 & $86.21 \%$ & $98.25 \%$ & $80.65 \%$ & $99.73 \%$ & $99.34 \%$ & $\begin{array}{c}\text { P } \\
\text { Rapid } \\
\text { Nonreactive }\end{array}$ \\
\hline & 58 & 2930 & 2938 & & & & & \\
\hline
\end{tabular}

SN: sensitivity Table number: 2); SP: specificity PPV; positive predictive value, NPV; negative predictive value; Chi-square $=2053.858$ with 1 degree of freedom . 
Int.J.Curr.Microbiol.App.Sci (2018) 7(1): 733-739

Fig.1 Distribution of the positive cases according to test

Rapid Diagnostic Test (502)

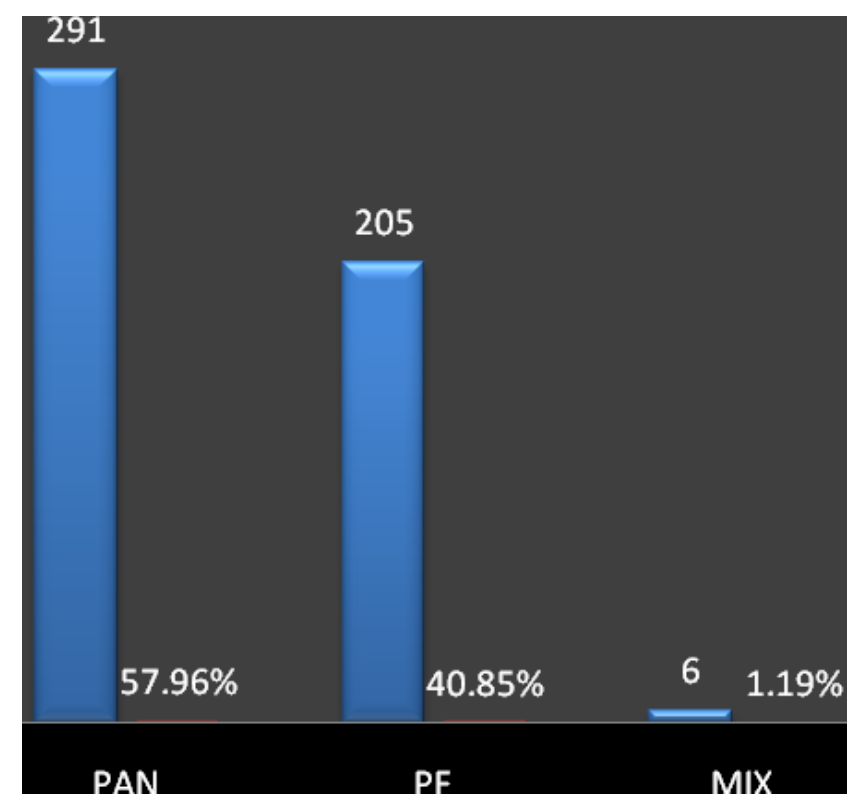

Slide examination (79)

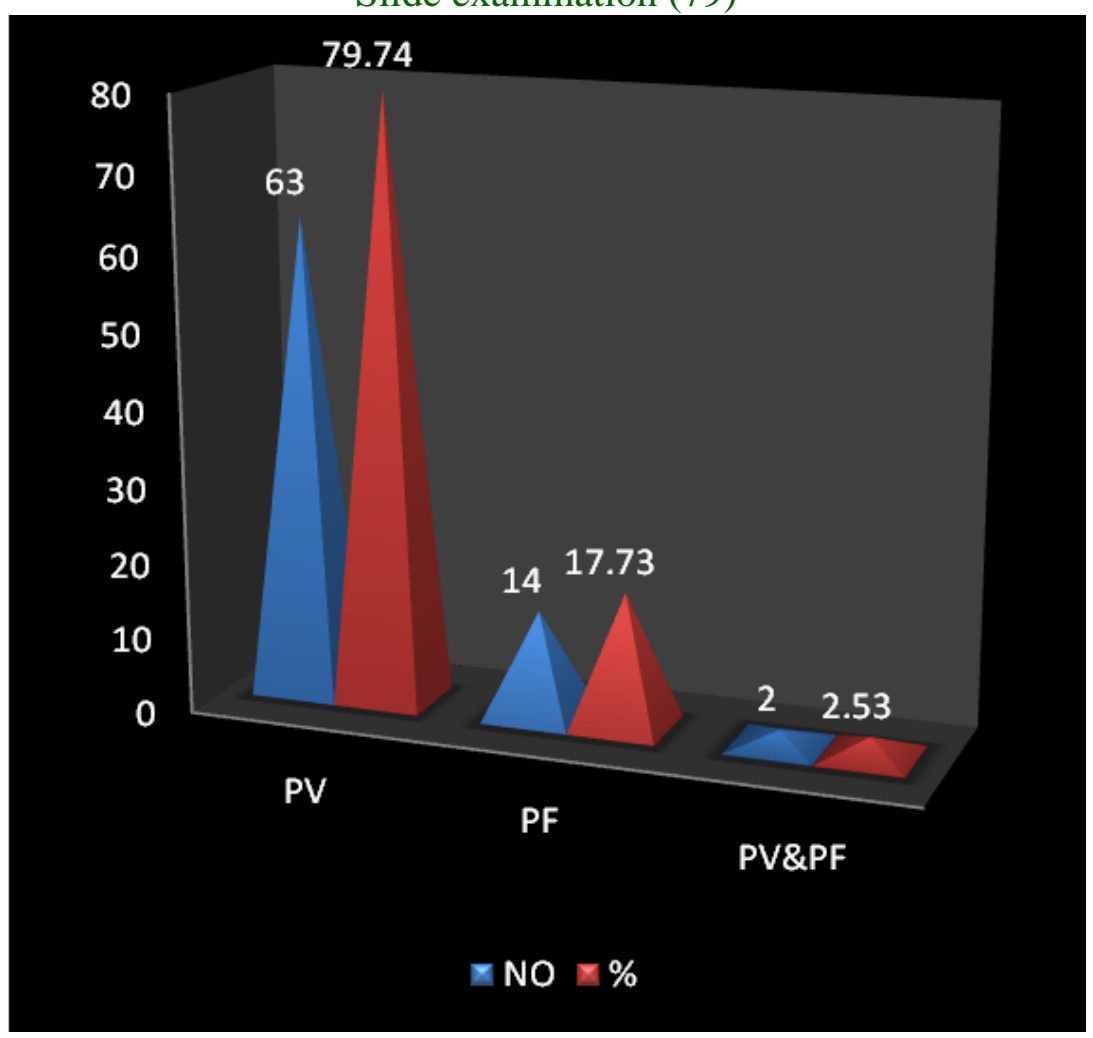


Fig.2 Seasonal distribution of positive cases

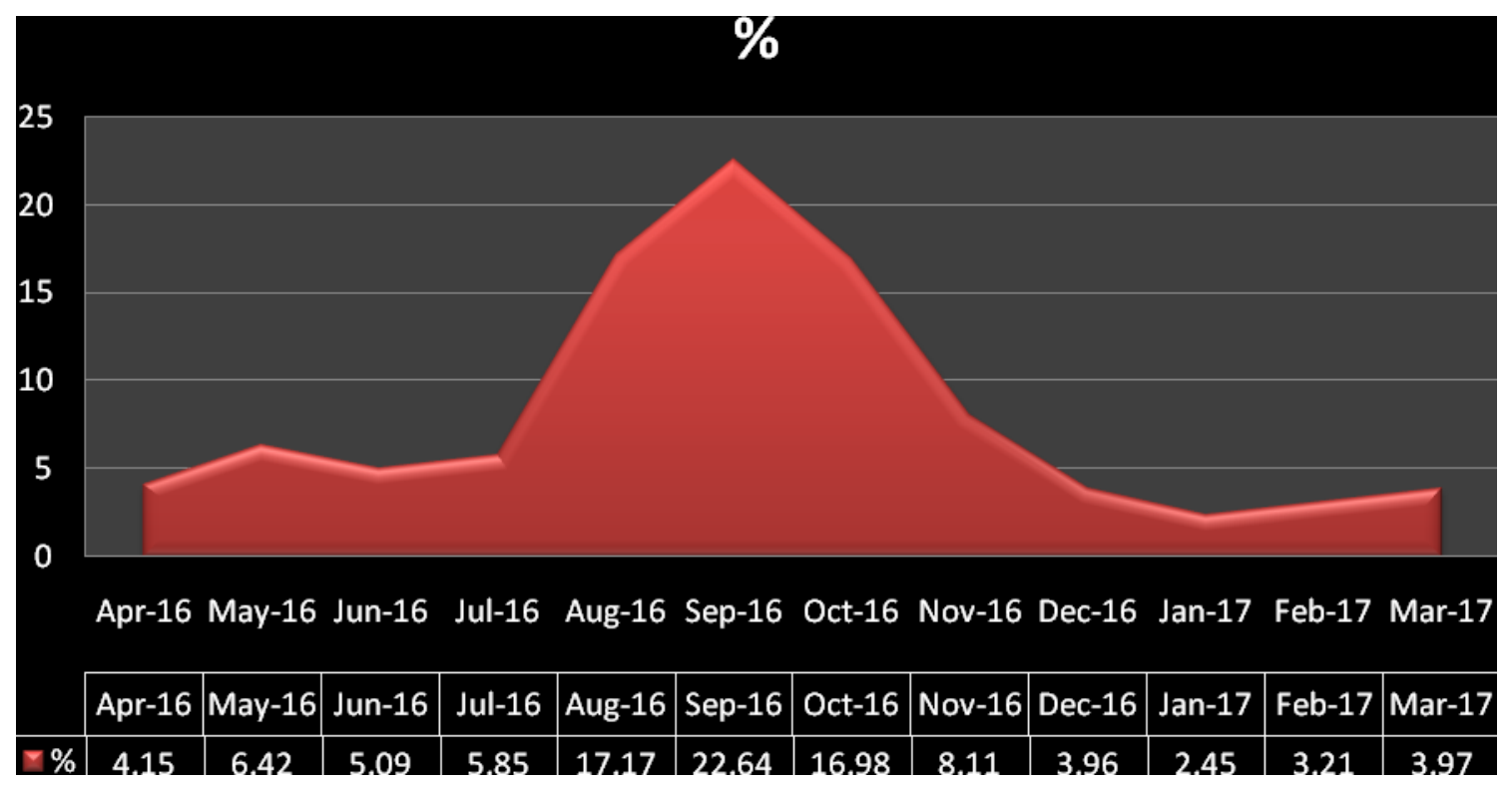

Also a study done by Nmadu et al., (2012) $64 \%$ cases were in the age group 1 to 10 years in Nigeria. This can be due to differences socio-economic conditions of patients, knowledge about healthcare and public health practices.

In the present study, Plasmodium falciparum, Plasmodium vivax and mixed species infection was $56.70 \%, 41.80 \% \& 01.50 \%$ respectively. Our study results were close to study done by Dharmendra Prasad Singh et al., (2016) whose result were $67 \%, 32 \%$ \& $1 \%$ respectively. However, Karlekar et al., (2012) from Gadchiroli reported Plasmodium vivax $33.80 \%$ and Plasmodium falciparum $66.60 \%$. This can be due to differences in geographical variation of the spp.

In this study cases were more in August, September and October month. Our results were similar to study done by Pankaj Gangwal et al., (2016) and Gurjeet Singh et al., (2015)). The high prevalence of malaria in this period could be due to collection of water in rainy season and mosquito breeding.

In our study Specificity and PPV of rapid diagnostic test were $99.60 \%, 80.60 \%$ and sensitivity and NPV were $86.2 \%, 99 \%$, our findings were consistent with the study done by Ruby Naz et al., (2016) which was $93.60 \%, 87.20 \%$ and $91.30 \%, 95.70 \%$ respectively. So, it is a good diagnostic and a screening test.

The severe illness and outbreaks emphasizes the importance of rapid diagnosis to decrease the related complications and thereby combating significant mortality and morbidity.

Our study revealed that the district still have the potential to suffer Malaria outbreak, if necessary interventions are not done for prevention and control of the disease. Early diagnosis and control activities well ahead of the pre-monsoon and monsoon season should target more to the communities with lower 
socio-economic status and the young age groups mostly males. Lower prevalence of malaria after 30 years of age could be attributed to development of immunity (resistance) due to clinical or subclinical exposures.

Rapid diagnostic test for diagnosis of malaria is as reliable and accurate as microscopy. Rapid diagnostic test use should be considered as more cost effective in the areas characterized by high-moderate intensity malaria transmission and in situations where health services are inadequate or absent.

\section{References}

Alireza Salimi Khorashad, Masoud Salehi; Bita Rosha. The Comparison of Microscopic Method and Rapid Diagnostic Test in Detecting Plasmodium species. Int $\mathbf{J}$ Infect. September 2014; 1(3): e21441.

Chatterjee KD; Parasitology (Protozoology and Helminthology), 13th edn. New Delhi: CBS, 2009; 90-127.

Dharmendra Prasad Singh, Rajesh Kumar Verma, Amit Singh et al., A retrospective study of malaria from western part of Uttar Pradesh, India int. J. Pharma. sc. Research,july201623205148.

EE Ayogu, CV Ukwe, EO Nna Assessing the reliability of microscopy and rapid diagnostic tests in malaria diagnosis in areas with varying parasite density among older children and adult patients in Nigeria. 2016. 62 (3): 150-156.

Estimation of True Malaria Burden in India
Fact Sheet: World Malaria Report, 2016.

Gurjeet Singh, AD Urhekar, Ujwala Maheshwari Prevalence of Malaria in a Tertiary Care Hospital in Navi Mumbai, India J Bacteriol Parasitol., 2015, 6:2.

Karlekar SR, Deshpande MM, Andrew RJ. Asymptomatic Plasmodium vivax and Plasmodium falciparum infections in tribal population of village in Gadchiroli District of Maharastra State, India. Biological Forum An int J. 2012; $4: 42-44$

Mengistu Hailemariam and Solomon Gebre. Trend analysis of malaria prevalence in Arsi Negell health center, Southern Ethiopia. J. Infec. Dis. Immune. 2015, 7(1)1-6.

Nmadu P. M., Peter E., Alexander P., Koggie A. Z., Maikenti J. I. The Prevalence of Malaria in Children between the Ages 2-15 Visiting Gwarinpa General Hospital Life-Camp, Abuja, Nigeria Journal of Health Science, 2015; 5(3): 47-51.

Paniker's textbook of medical parasitology, $8^{\text {th }}$ edition, New Delhi, JAYPEE, Pp. 66-68.

Pankaj R Gangwal, N A Razvi Seasonality of malaria in and around Aurangabad district, Marathwada region of Maharashtra, India Int. Medical Journal, 3(7), July 2016, 620-623.

Ruby Naz, Sameena khan, Mohammad Khalid Farooqui Comparison of microscopic determination and rapid diagnostic tests (rdts) in the Detection of Plasmodium Infection Sch. J. App. Med. Sci., 2016; 4(7D): 2539-2543.

\section{How to cite this article:}

Ranweer, Jyotsna Chandwani, Seema Gupta, Geeta Parihar and Geeta Pachori. 2018. Diagnostic Vision and Association with Demographics in Malaria Patients. Int.J.Curr.Microbiol.App.Sci. 7(01): 733-739. doi: https://doi.org/10.20546/ijcmas.2018.701.088 